\title{
Sistematização da Assistência de Enfermagem ao portador de Diabetes Mellitus e Insuficiência Renal Crônica
}

\author{
Systematization of Nursing Assistance to patients with Diabetes Mellitus and Chronic Renal Insufficiency
} Sistematización de la Asistencia de Enfermería al portador de Diabetes Mellitus y Insuficiencia Renal Crónica

\author{
Nildo Batista Mascarenhas, Álvaro Pereira, Rudval Souza da Silva, Mary Gomes da Silva' \\ 'Universidade Federal da Bahia. Escola de Enfermagem. Salvador, BA
}

Submissão: 1 1/01/2009

Aprovação: 26/09/2010

\section{RESUMO}

Tratou-se de um estudo de caso clínico desenvolvido durante as atividades práticas da disciplina Enfermagem Clínico-Cirúrgica I, do curso de Graduação em Enfermagem de uma universidade pública do Estado da Bahia, Que objetivou relatar a aplicação da Sistematização da Assistência de Enfermagem na assistência a um paciente portador de Diabetes Mellitus e Insuficiência Renal Crônica. Com o desenvolvimento do estudo, em especial após a melhora positiva da paciente face às condutas planejadas e implementadas e, considerando as reflexões Que emergiram, foi possível constatar a necessidade da interface entre a Sistematização da Assistência de Enfermagem, equipe de enfermagem e paciente no processo do cuidar, frente à excelência e singularidade dos cuidados de enfermagem.

Descritores: Diabetes mellitus; Insuficiência renal; Processos de enfermagem; Cuidados de enfermagem.

\section{ABSTRACT}

This is a clinical case study developed during the practical activities of the discipline Surgical Clinical Nursing I, of course of Graduation in Nursing of a public university of Bahia State, that aimed to report the application of the Systematization of Nursing Assistance in the assistance to a client whit Diabetes Mellitus and Chronic Renal Insufficiency. With the development of the study, especially after the positive improvement of the client, face to assistance planned and implemented and considering the reflections that emerged, it was possible to evidence the need for interface between Systamatization of Nursing Assistance, the nursing staff and client in the care process, at the excellence and uniQueness of nursing care.

Key words: Diabetes mellitus; Renal insufficiency; Nursing process; Nursing care.

\section{RESUMEN}

Este es un estudio de caso desarrollado durante las actividades prácticas de la disciplina de Enfermería Clínica Quirúrgica I, del curso de Grado en Enfermería en una universidad pública del Estado de Bahía, Que tuvo por objetivo relatar la aplicación de la Sistematización de la Asistencia de Enfermería (SAE) en asistencia a un paciente conducir a la Diabetes Mellitus y la Insuficiencia Renal Crónica. Con el desarrollo del estudio, sobre todo después de la mejora positiva del paciente, se enfrentan para llevar a cabo y planifieuen y ejecuten teniendo en cuenta las ideas que surgieron, es posible ver la necesidad de interfaz entre la Sistematización de la Asistencia de Enfermería y el personal de enfermería y el paciente en el proceso de atención, en la excelencia y la singularidad de los cuidados de enfermería.

Descriptores: Diabetes mellitus, Insuficiencia renal; Procesos de enfermería; Atención de enfermería.

AUTOR Nildo Batista Mascarenhas. Rua Dr. Renato Mendonça, 308 - apto 703. CEP 40285-440. Salvador, BA.

CORRESPONDENTEＥ-mail: nildomascarenhas@gmail.com 


\section{INTRODUÇÃO}

O Diabetes Mellitus (DM) é um grupo de doenças metabólicas caracterizada por níveis elevados de glicose no sangue (hiperglicemia), decorrentes de defeitos na secreção e/ou na ação da insulina. A insulina, hormônio produzido pelo pâncreas, tem o papel de controlar o nível de glicose no sangue, ajustando a produção e armazenamento de glicose $\mathrm{e}^{(1)}$. A prevalência mundial do DM apresentou um aumento significante nas últimas duas décadas e, como consequência, o aumento no número de indivíduos diabéticos, devido a fatores como: envelhecimento populacional, níveis crescentes de obesidade e sedentarismo ${ }^{(2)}$.

Existem tipos distintos de diabetes, causados por uma complexa interação de fatores genéticos, ambientais e de estilo de vida. Neste contexto, "a desregulação metabólica associada ao diabetes pode gerar alterações fisiopatológicas secundárias em múltiplos sistemas orgânicos, impondo um enorme 'fardo' sobre o indivíduo com diabetes e o sistema de saúde" (3).

Em pessoas portadoras deste distúrbio, por um período de tempo consideravelmente longo - em média dez anos, o DM pode causar inúmeras doenças crônicas como a insuficiência renal crônica, uma enfermidade Que se caracteriza por lesão renal e perda progressiva e irreversível da função dos rins. Em sua fase mais avançada ela é denominada de Insuficiência Renal Crônica (IRC), visto Que os rins não conseguem manter a homeostase do indivíduo ${ }^{(4)}$.

Considerando Que o DM e a IRC são importantes problemas de saúde pública, o enfermeiro ao prestar cuidados à pessoas portadoras destas afecções, tanto na atenção básica Quanto no âmbito hospitalar, vê-se frente a um grande desafio - sistematizar o cuidado. Assim sendo, a Sistematização da Assistência de Enfermagem (SAE), neste contexto, emerge como instrumento essencial Que pode subsidiar e/ou guiar a assistência de enfermagem, com foco na integralidade das dimensões do ser cuidado e garantindo ao enfermeiro, autonomia e segurança, além de atendimento humanizado e individual. Sendo assim, este estudo objetiva relatar a aplicação da SAE no cuidado a um paciente portador de DM e IRC.

O DM não representa uma única patologia, mas um grupo heterogêneo de distúrbios metabólicos Que apresentam em comum a hiperglicemia ${ }^{(2)}$. Os fatores Que contribuem para a hiperglicemia dependem da etiologia do DM e incluem redução da secreção de insulina, diminuição da utilização e/ou aumento da produção de glicose $^{(1)}$. Desta forma, pode-se inferir que o diabetes é classificado segundo o processo patogênico Que acarreta a hiperglicemia ${ }^{(3)} \mathrm{e}$ as duas categorias gerais de classificação do diabetes são designadas como tipos 1 e 2.

O DM tipo I (DMI), forma presente em 5-10\% dos casos, caracteriza-se pela destruição das células beta pancreáticas com a consequente deficiência relativa ou absoluta de insulina. Na maior parte dos casos, a destruição destas células é mediada por autoimunidade, existindo, porém, casos em Que não há evidências de processo autoimune, sendo referida, portanto, como forma idiopática do DMI. Além dos fatores imunológicos, os fatores ambientais (como vírus e toxinas) e genéticos, têm sido considerados como desencadeadores do DM I ${ }^{(2)}$.

O DM tipo 2 (DM2), forma presente em $90-95 \%$ dos casos diagnosticados, caracteriza-se por defeitos na ação da insulina (resistência à insulina) e na sua secreção. A maior parte das pessoas com essa forma de diabetes apresenta sobrepeso ou obesidade. $\mathrm{O}$ DM2 geralmente é diagnosticado após os 40 anos, porém pode ocorrer em QualQuer idade ${ }^{(2)}$.

As manifestações clínicas mais frequentes e comuns a ambos são: poliúria, polidipisia, polifagia, fadiga, irritabilidade, feridas cutâneas Que demoram de cicatrizar, turvação visual, formigamento nas mãos e nos pés e alterações visuais súbitas.

Com relação ao tratamento deste distúrbio, pode-se dizer que a sua meta principal consiste em normalizar a atividade da insulina e os níveis sanguíneos de glicose, a fim de prevenir o desenvolvimento de complicações cardiovasculares e neuropáticas e os cinco componentes essenciais da terapêutica são: tratamento nutricional, exercícios físicos, monitorização, terapia farmacológica e educação $^{(1)}$.

O enfermeiro tem papel essencial na prestação de cuidados a indivíduos com DM, principalmente por desenvolver atividades educativas, com o objetivo de aumentar o nível de conhecimento dos pacientes e comunidade, além de contribuir para a adesão destes ao tratamento. Assim, o enfermeiro, estando mais próximo e capacitado para o desenvolvimento das atividades educativas efetivas, muito poderá fazer para o controle desta doença e para a promoção da saúde deste grupo ${ }^{(5)}$ Nesta perspectiva, é correto inferir Que as ações educativas, desenvolvidas juntamente com o paciente, família e comunidade, têm um papel fundamental no controle dessa enfermidade, já Que as complicações oriundas do diabetes estão diretamente relacionadas ao conhecimento para o autocuidado diário e ao estilo de vida saudável(6).

Para os pacientes diagnosticados com o DMI o uso regular da insulina, associado a exercícios físicos e uma dieta adequada, equilibrada e rigorosa, torna-se essencial para o controle da glicemia, já aQueles recém diagnosticados com o DM2, em geral, adota-se inicialmente a prática regular de exercícios físicos, redução de peso (para pacientes obesos) e uma reeducação alimentar. Caso essas medidas não obtenham êxito, o uso de hipoglicemiantes orais é indicado e a insulinoterapia é iniciada Quando os tratamentos anteriores não produzem resultados satisfatórios e o controle da glicemia não é efetivo. Além dessas medidas, nos casos de pacientes com obesidade, a cirurgia bariátrica tem sido discutida como alternativa futura de solução para o DM2.

Com base no exposto, pode-se afirmar Que o diagnóstico tardio do diabetes mellitus pode acarretar consequências agudas e crônicas, devido à exposição do indivíduo à hiperglicemia por um longo período de tempo (cerca de 10 a 15 anos).

Dentre as complicações agudas, destacamos a cetoacidose diabética. Esta é uma complicação típica do DMI, causada por uma Quantidade inadequada de insulina, constituindo-se em uma afecção grave em Que a hiperglicemia e a hiperosmolaridade predominam, com alterações no sensório (sensação de consciência), ocorrendo frequentemente em pessoas idosas. Esse déficit de insulina resulta em distúrbios no metabolismo dos carboidratos, proteínas e lipídios. Os três principais aspectos clínicos nesta complicação são a hiperglicemia, desidratação com perda de eletrólitos e acidose metabólica ${ }^{(1)}$.

As complicações crônicas do DM estão se tornando cada vez mais comuns e evidentes na medida em Que, cada vez mais as pessoas desenvolvem este distúrbio. No mundo, a estimativa de 
óbitos atribuída ao DM encontra-se em torno de 800 mil. Contudo, salienta-se Que esse número é consideravelmente subestimado, pois frequentemente, na declaração de óbito, não é mencionado o DM como causa da morte e sim as suas complicações, particularmente as cardiocerebrovasculares ${ }^{(2)}$.

Dentre as complicações crônicas do DM, vale destacar a nefropatia, atualmente considerada como a principal causa de $\operatorname{IRC}^{(7)}$, condição esta tida como irreversível. A IRC é uma síndrome clínica causada pela perda progressiva e irreversível das funções renais, resultando em uremia $^{(8)}$, cujo tratamento é bastante invasivo e incômodo.

A IRC é uma doença com vários efeitos na vida do pacientes e de difícil tratamento, com sérias implicações físicas, psicológicas e sócioeconômicas, não apenas para o indivíduo, mas também para a família e a comunidade ${ }^{(9)}$. Além disso, a IRC é uma enfermidade Que altera o cotidiano do indivíduo que a vivencia, sendo caracterizada também como um problema social, Que interfere no papel Que esse indivíduo desempenha na sociedade. Então, estabelecese um longo processo de adaptação a essa nova condição, no Qual o indivíduo precisa identificar meios para lidar com o problema renal e com todas as mudanças e limitações Que o acompanham ${ }^{(10)}$.

Mediante estes fatos, podemos inferir Que o estresse da doença renal afeta a autoestima, as relações familiares e conjugais, além de Quase todos os aspectos da vida diária do indivíduo acometido por esta complicação crônica consequência do diabetes mellitus. À medida Que a função renal diminui e o paciente evolui para a IRC, ele apresenta em geral falência de múltiplos órgãos levando a sequelas como, por exemplo, deficiência da acuidade visual, impotência e insuficiência cardíaca ${ }^{(1)}$.

Sendo assim, é necessário e fundamental julgar as respostas dessa clientela aos cuidados de enfermagem. O enfermeiro tem um papel essencial no cuidado aos indivíduos portadores de IRC e DM, principalmente no Que tange ao estímulo ao autocuidado à saúde, de modo a facilitar a cooperação e adesão do paciente ao tratamento, além de estimulá-lo a enfrentar as mudanças cotidianas e a alcançar o seu bem-estar (II). Esta situação nos reporta a Sistematização da Assistência de Enfermagem, a Qual contribui para a organização do trabalho do enfermeiro e para um melhor relacionamento deste com o paciente, proporcionando assim melhor norteador do cuidado prestado pelo enfermeiro a essa clientela"(9).

A consulta de enfermagem representa o primeiro momento para a aplicação da SAE, sendo uma atividade privativa do enfermeiro, Que através de um método e estratégia de trabalho científico, realiza a identificação das situações de saúde/doença, subsidiando a prescrição e implementação das ações de Enfermagem. Dessa forma, vem contribuir para a promoção, prevenção, recuperação e reabilitação da saúde do indivíduo, família e comunidade ${ }^{(12)}$. Neste contexto, a SAE fornece um método organizado e sistemático para uma análise do estado de saúde do indivíduo, identificando suas necessidades e padrões de resposta aos problemas, possibilitando a determinação de soluções apropriadas no atendimento dessas necessidades ${ }^{(13)}$.

Ressalta-se Que na aplicação da SAE se faz necessário entender o paciente como uma pessoa Que age, reage e interage diferentemente, à medida Que sua situação particular de vida muda, ao longo do ciclo vital. Ao interagir com seres humanos, nenhuma ação instrumental, por mais aperfeiçoada Que seja, pode estar desvinculada dos aspectos humanísticos e de suas necessidades espirituais Que lhes são intrínsecas ${ }^{(14)}$

Diante do exposto, é possível depreender que o papel da enfermagem na assistência ao paciente portador de DM e IRC é essencial, principalmente através de um cuidado de enfermagem sistematizado e coerente, com enfoeue no ser humano e na sua família, de modo a tornar menos estressante e doloroso o tratamento desta complicação secundária a este distúrbio plurimetabólico denominado diabetes mellitus.

\section{METODOLOGIA}

Tratou-se de um estudo de caso clínico, Que se constitui em uma modalidade de pesquisa bastante utilizada nas ciências biomédicas e sociais, comumente realizado no intuito de se reconhecer situações específicas. O objetivo do estudo clínico é contribuir para a compreensão de determinadas situações e a conseQuente aplicação de condutas compatíveis, vindo a constituir-se de um importante instrumento de investigação das Questões enfrentadas por profissionais em sua prática ${ }^{(15)}$.

O presente estudo foi realizado em maio de 2008, em uma unidade de internação de Clínica Médica de um hospital público da cidade de Salvador, BA, durante as atividades práticas da disciplina Enfermagem Clínico-Cirúrgica I. O sujeito escolhido para o estudo foi uma idosa de 81 anos, portadora de DM e IRC Que foi acompanhada durante o período de admissão até sua alta hospitalar.

A paciente aceitou participar espontaneamente do estudo, tendo assinado o termo de autorização no ato da internação, orientada sobre a realização do mesmo e sobre a confidencialidade das informações, conforme o preconizado pela Resolução 196/96 do Conselho Nacional de Saúde, Que se refere aos aspectos éticos para pesquisas que envolvem seres humanos.

Para a coleta dos dados foi utilizado um impresso do histórico de enfermagem composto de anamnese e exame físico, baseado na Teoria das Necessidades Humanas Básicas proposta por Wanda Horta, utilizado durante as atividades práticas da disciplina onde se desenvolveu esse estudo. Este histórico estava dividido em duas partes: a primeira, composta por dados sobre as principais características sócio-demográficas da paciente e a segunda por Questões semi-estruturadas sobre as necessidades humanas básicas.

A aplicação do histórico de enfermagem possibilitou o levantamento de informações de ordem subjetiva e individual sobre os aspectos socioculturais e das necessidades humanas básicas da paciente. $\mathrm{O}$ exame físico possibilitou complementar a coleta de dados, fornecendo deste modo, os dados objetivos Que subsidiaram a definição dos problemas, estabelecimentos dos diagnósticos, das intervenções e evolução de enfermagem.

Após a análise dos dados coletados, levantaram-se os problemas de enfermagem Que subsidiaram a definição dos Diagnósticos de Enfermagem, segundo a Taxonomia II da North American Nursing Diagnosis Association (NANDA) ${ }^{(16)}$. Posteriormente, planejaramse as intervenções de acordo com os problemas identificados na paciente em estudo, centrando nossa atenção na integralidade da assistência durante o processo do cuidar e dos cuidados de enfermagem. A utilização de um modelo de coleta voltado para os grandes domínios e classes do referencial da NANDA poderia ter facilitado o trabalho de definição dos diagnósticos, o Que se constituiu numa das limitações do estudo, considerando a utilização 
do formulário padrão do Hospital, o Qual não esta adequado a estrutura de domínios e classes da Taxonomia II da NANDA.

\section{RESULTADOS E DISCUSSÃO}

\section{Apresentação do Caso}

I.R.G, 81 anos, sexo feminino, negra, brasileira, natural de Salvador, BA, viúva, aposentada, primeiro grau incompleto, recorreu ao hospital por conta de dor intensa em flanco esQuerdo à esclarecer. DM 2, hipertensa, dislipidêmica, portadora de IRC. Mobilidade no leito prejudicada por conta de dor em flanco esquerdo, vigil, padrões de sono e repouso alterados, com Queixa de nictúria e da rotina da unidade. Ao exame apresentou couro cabeludo íntegro, mucosas oculares normocrômicas, escleróticas anictéricas, acuidade visual e auditiva diminuídas, narinas sem lesões. Tórax simétrico, murmúrio vesicular fisiológico em ambos hemitórax; bulhas normofonéticas em 2 tempos; abdome distendido, doloroso à palpação, principalmente no flanco esQuerdo; RHA presentes; extremidades perfundidas e sem edemas. Definiu-se como uma pessoa tranquila e "sem grandes preocupações com a sua doença". Descobriu Que era diabética há 26 anos após a realização de uma cirurgia de hérnia. Sua última hospitalização foi no dia 23/06/07, para a realização de uma cirurgia de catarata, a Qual ocorreu sem intercorrências. Apresentou bom conhecimento acerca do diabetes e dos mecanismos de controle da mesma. Consulta-se com o nefrologista a cada dois meses. Não pratica atividades físicas. Conforme a prescrição médica, estava em uso das seguintes medicações: insulina NPH SC pela manhã; AAS 100 mg após o almoço; Atensina 0,150 pele a manhã e pela noite; Sinvastatina 20mg à noite, Carbonato de Cálcio pela manhã e I ampola de furosemida EV pela manhã e soroterapia em acesso periférico no MSD. Negou tabagismo, alcoolismo e alergias. Apetite e paladar preservados, deglutição e mastigação realizada à custa de prótese dentária total. Diurese presente com relato de poliúria assim como evacuações ausentes há \pm 4 dias. Referiu ainda não ingerir muito líeuido ao dia "para evitar ficar urinando toda hora". Cansaço ao vestir-se, e enjôo ao andar por conta da labiritite, o que a deixou restrita Quanto à deambulação, permanecendo por muito tempo deitada no leito. Segundo a filha Que a acompanhava no momento da realização do histórico de enfermagem, a paciente alimenta-se fora do horário e por conta disso ficava sem apetite e sem almoçar. Além disso, a filha relatou também que a paciente passava a maior parte do tempo deitada no sofá de sua casa e tinha "preguiça" de deambular e praticar atividades físicas. Dados vitais e antropométricos: PA: 150x80 mmHg; FC: $80 \mathrm{bpm}$; FR: 16 inc/min; T: 36,5 ${ }^{\circ}$; Peso: $65,7 \mathrm{~kg}$; Altura: 1,59m; IMC: 25,99.

\section{Problemas identificados e Diagnósticos de Enfermagem tendo por base a Taxonomia II da NANDA}

A partir do levantamento de problemas e demandas de cuidados, identificamos e estabelecemos nove Diagnósticos de Enfermagem relacionados às dimensões biopsicossociais da paciente. A seguir (Apêndice 1) descrevemos os problemas identificados e os respectivos Diagnósticos de Enfermagem.

\section{Planejamento da Assistência de Enfermagem}

Com base nos problemas identificados e na formulação dos diagnósticos apropriados à situação da paciente, fez-se necessário planejar uma assistência de enfermagem adequada à sua individualidade, de modo a garantir Que não somente a situação clínica seja superestimada, mas Que os aspectos biopsicossociais assumam posição privilegiada nas intervenções de enfermagem implementadas. Deste modo, propomos (Apêndice 2) as seguintes intervenções, com as suas respectivas justificativas:

Após o planejamento e implementação das condutas apresentadas na prescrição de enfermagem é possível afirmar Que as respostas da paciente face às intervenções adotadas foram positivas. Na primeira semana, percebeu-se uma evolução pouco significativa do seu Quadro clínico, considerando-se que ainda Queixava-se de dor intensa em flanco esQuerdo e constipação.

Na segunda semana, a evolução da paciente foi um pouco mais significativa, porém a dor e constipação persistiram apesar de menos intensas e a mobilidade física ainda continuava reduzida. Com isso, optou-se por aumentar os estímulos à deambulação, auxiliando a mesma a sentar-se no leito e em seguida deambular por 15 minutos, pelo menos duas vezes ao dia, de modo compatível com as suas limitações.

Na terceira semana, observou-se Que a paciente evoluiu com uma resposta positiva e a dor, apesar de ainda não ter sido completamente eliminada, houve uma redução significativa. A deambulação, bastante estimulada na semana anterior, tornou-se mais constante, superando o diagnóstico de mobilidade física reduzida, identificado no início da hospitalização. Não se registraram episódios de hipoglicemia e as evacuações tornaram-se mais frequentes.

Vale ressaltar Que apesar da evolução satisfatória da paciente, observada diariamente pelos autores deste estudo, e da eficácia das intervenções implementadas, era notório Que a equipe de enfermagem da unidade não considerava devidamente o Que havia sido planejado para a paciente. A ênfase nos procedimentos técnicos e na prescrição médica impossibilitou Que as condutas planejadas fossem por completo adotadas. Prova disso, foi Que a inspeção diária da pele, a hidratação da mesma e o auxílio e estímulo à deambulação não foram observados e/ou efetivados por outros membros da equipe de enfermagem da clínica onde esse estudo foi desenvolvido. A cooperação da equipe, apesar de fundamental, não foi suficiente para Que a paciente tivesse um resultado ainda mais satisfatório, como era esperado pelos autores durante a formulação das prescrições de enfermagem.

Apesar dos problemas identificados na implementação total da prescrição de enfermagem, obteve-se êxito na assistência prestada à paciente, em especial na redução da constipação, na melhora da mobilidade física e no seu estado geral.

\section{CONSIDERAÇÕES FINAIS}

Ao final dessa experiência pudemos perceber que a paciente hospitalizada, portadora de diabetes mellitus e de insuficiência renal crônica, necessitou de condutas eficazes Que visaram não somente o controle dos desequilíbrios na dimensão fisiopatológica, mas também com enfoque nos aspectos psicossocioespirituais. A SAE, considerada como um método científico orienta a prática do enfermeiro e de toda sua equipe, sendo de extrema importância para Que o cuidado profissional de enfermagem prestado ao paciente hospitalizado seja eficiente e individualizado, de modo a garantir a integralidade e a Qualidade da assistência. 
Com isso, a realização do histórico de enfermagem Que priorizou a totalidade da atenção ao paciente, possibilitando a identificação dos problemas, formulação de diagnósticos de enfermagem precisos, o planejamento adequado e avaliação diária das intervenções realizadas, foi essencial para a recuperação e reabilitação do sujeito do estudo.

As respostas da paciente face às condutas implementadas $\mathrm{e}$ conforme o planejado foram positivas, corroborando a eficiência da assistência prestada e o alcance dos objetivos propostos. Porém, evidenciaram-se como necessárias, durante a realização do estudo, uma maior integração da eQuipe de Enfermagem e a superação do tecnicismo vinculado ao modelo biomédico reducionista, Que acaba afastando a enfermagem da essência de sua prática: o cuidado.

A implementação da SAE exige da profissão uma integração efetiva com a equipe multidisciplinar, ao considerá-la um instrumento essencial ao bom desenvolvimento das ações do enfermeiro e, principalmente, na adoção de ações individualizadas e humanizadas. Como alternativas para promover a adoção efetiva da SAE, propomos uma maior aproximação da equipe de enfermagem com a SAE, através do conhecimento e o fomento a discussões sobre sua implementação; superação do tecnicismo Que ainda é hegemônico na assistência Que a enfermagem presta aos indivíduos e, por fim a integração e prática do cuidado humanizado e sistematizado por toda a equipe multidisciplinar.

Esperamos com este relato fomentar a pesquisa e o debate acerca da temática e demonstre a importância da SAE para a prática profissional do enfermeiro e de toda a equipe de saúde, em especial para os pacientes Que apresentam enfermidades como a IRC e DM, muito incidentes nas unidades de internação dos serviços públicos, e que exigem da enfermagem condutas rigorosas e efetivas.

\section{REFERÊNCIAS}

I. Smeltzer SC, Bare BG. Brunner \& Suddarth. Tratado de Enfermagem Médico-Cirúrgica. $10^{\mathrm{a}}$ ed. Rio de Janeiro: Guanabara Koogan; 2005.

2. Sociedade Brasileira de Diabetes. Tratamento e Acompanhamento do Diabetes Mellitus- Diretrizes da Sociedade Brasileira de Diabetes. Rio de Janeiro: Diagraphic Editora; 2007.

3. Powers AC. Diabetes Melito. In: Harrison TR. Medicina Interna. $16^{\mathrm{a}}$ ed. Rio de Janeiro: McGraw-Hill; 2006.

4. Romão Júnior JE. Doença renal crônica: definição, epidemiologia e classificação. I Bras Nefrol [periódico online] 2004 Ago [citado em: 02 mai 2009]; 26(3). Disponível em: http://www.jbn.org.br/ 26-3 I/v26e3s I p00 I.pdf

5. Tavares DMS, Rodrigues RAP. Educação conscientizadora do idoso diabético: uma proposta de intervenção do enfermeiro. Rev Esc Enferm USP 2002; 36(1): 88-96.

6. Faeda A, Leon CGRMP. Assistência de enfermagem a um paciente portador de Diabetes Mellitus. Rev Bras Enferm 2006; 59(6): 818-21.

7. Lenardt MH, Hammerschmidt KSA, Borghi ACS, Vaccari E, Seima MD. O idoso portador de nefropatia diabética e o cuidado de si. Texto Contexto Enferm 2008; 17(2): 313-20.

8. Cendoroglo M, Sardenberg C, Suassuna P. Insuficiência renal crônica: etiologia, diagnóstico e tratamento. In: Schor N, Srougi M. Nefrologia, urologia clínica. São Paulo: Sarvier; 1998. p. 29-41.

9. Lata AGB, Albueuereue IG, Carvalho LASBP, Lira ALBC. Nursing diagnosis in adults on hemodialysis. Acta Paul Enferm

2008; 2 I (supl): 1 60-3.

10. Reis CK, Guirardello EB, Campos CIGI. O indivíduo renal crônico e as demandas de atenção. Rev Bras Enferm 2008; 6 l (3): 336-4l.

I I. Pacheco GS, Santos I, Bregman R. Características de clientes com doença renal crônica: evidências para o ensino do autocuidado. Rev enferm UERI 2006; I 4(3): 434-9.

12. Conselho Federal de Enfermagem (BR). Resolução n 358/ 2009. Dispõe sobre a Sistematização da Assistência de Enfermagem e a implementação do Processo de Enfermagem em ambientes, públicos ou privados, em Que ocorre o cuidado profissional de Enfermagem, e dá outras providências. [citado em: 25 nov 2009]. Rio de Janeiro; 2009. Disponível em: http:/ /www.portalcofen.com.br

13. Sampaio LABN, Pellizzetti N. Método Científico - Instrumento Básico da Enfermeira. In: Cianciarullo TI, organizadora. Instrumentos Básicos para o Cuidar - um desafio para a Qualidade de assistência. São Paulo (SP): Atheneu; 2007. p. 25-38

14. Silva RS, Santos MHER. Sistematização da Assistência de Enfermagem como uma estratégia para a autonomia do enfermeiro. Nursing 2009; 36(12): 435-42.

15. Gil AC. Como Elaborar Projetos de Pesquisa. 4ª ed.São Paulo (SP): Atlas; 2006.

16. North American Nursing Diagnosis Association. Diagnósticos de Enfermagem da NANDA: definições e classificação $2007-$ 2008. Porto Alegre: Artmed; 2007. 


\begin{tabular}{|c|c|c|c|}
\hline Problemas identificados & $\begin{array}{l}\text { Diagnósticos de } \\
\text { enfermagem }\end{array}$ & Fatores relacionados & Características definidoras \\
\hline Sono e repouso alterados & $\begin{array}{l}\text { Padrão de sono } \\
\text { perturbado }\end{array}$ & Despertar frequente durante a noite, secundário à nictúria & $\begin{array}{l}\text { Dificuldade para adormecer e } \\
\text { sonolência matinal }\end{array}$ \\
\hline Enjôo ao deambular & Náusea & Irritação gástrica secundária à labirintite & $\begin{array}{l}\text { Relato de náusea e mal estar no } \\
\text { estômago }\end{array}$ \\
\hline Poliúria e nictúria & $\begin{array}{l}\text { Eliminação urinária } \\
\text { prejudicada }\end{array}$ & $\begin{array}{l}\text { Diminuição da capacidade da bexiga secundária à } \\
\text { glicosúria }\end{array}$ & Queixa de poliúria e nictúria \\
\hline Perda excessiva de líeuidos & $\begin{array}{l}\text { Risco de volume de } \\
\text { lípuidos deficiente }\end{array}$ & $\begin{array}{l}\text { Perdas excessivas por vias normais relacionado ao uso de } \\
\text { medicações (diuréticos) }\end{array}$ & \\
\hline $\begin{array}{l}\text { Ausências de evacuações } \\
\text { há } \pm 4 \text { dias }\end{array}$ & Constipação & $\begin{array}{l}\text { Atividade física insuficiente, hábitos de evacuações } \\
\text { irregulares, terapia farmacológica (carbonato de cálcio e } \\
\text { diuréticos), hábitos alimentares deficientes, motilidade do } \\
\text { trato gastrointestinal diminuída }\end{array}$ & $\begin{array}{l}\text { Abdome distendido, dor abdominal, } \\
\text { esforço para evacuar, freeuência } \\
\text { diminuída, fezes duras e secas, } \\
\text { ruídos intestinais hipoativos }\end{array}$ \\
\hline Dor no flanco esQuerdo & Dor crônica & Capacidade física crônica & Relato verbal de dor \\
\hline Cansaço & Intolerância à atividade & $\begin{array}{l}\text { Aumento das demandas metabólicas, secundário ao DM e } \\
\text { inatividade secundária a estilo de vida sedentário }\end{array}$ & $\begin{array}{l}\text { Relato verbal de astenia muscular e } \\
\text { desconforto aos esforços }\end{array}$ \\
\hline Mobilidade no leito & $\begin{array}{l}\text { Risco para mobilidade } \\
\text { física prejudicada }\end{array}$ & $\begin{array}{l}\text { Dor e intolerância à atividade, dor, desconforto, estilo de } \\
\text { vida sedentário, medicamentos }\end{array}$ & \\
\hline $\begin{array}{l}\text { Permanece muito tempo } \\
\text { deitada no leito e } \\
\text { "preguiça" de deambular e } \\
\text { praticar atividades físicas }\end{array}$ & Estilo de vida sedentário & $\begin{array}{l}\text { Conhecimento inadequados dos benefícios da atividade } \\
\text { física à saúde, falta de interesse, falta de motivação }\end{array}$ & $\begin{array}{l}\text { Escolha de uma vida diária sem } \\
\text { exercícios físicos, verbaliza } \\
\text { preferência por atividades com } \\
\text { pouco exercício físico }\end{array}$ \\
\hline
\end{tabular}

\section{Apêndice 1. Problemas identificados a partir dos achados do Histórico de Enfermagem e os Diagnósticos de Enfermagem levantados.}

\begin{tabular}{|c|c|}
\hline Prescrições propostas & Justificativas \\
\hline Monitorar o horário das refeições & $\begin{array}{l}\text { Para o paciente diabético e em uso de hipoglicemiantes orais e/ou insulina, uma refeição adequada e no horário } \\
\text { correto, minimiza os riscos de se desenvolver uma hipoglicemia. A fim de evitar que haja o desenvolvimento de uma } \\
\text { hipoglicemia, por alimentação inadequada e horários irregulares, foi necessário monitorar e certificar-se que a } \\
\text { paciente estava se alimentando no horário previsto e adequadamente }\end{array}$ \\
\hline Estimular a ingesta hídrica adequada & $\begin{array}{l}\text { Conforme exposto no histórico de enfermagem, a paciente não ingeria muito líQuido durante o dia, o que é tido como } \\
\text { um fator de risco considerando as necessidades diárias de ingesta hídrica, além do uso de diuréticos. Caso a ingestão } \\
\text { de líQuidos continuasse menor do que a eliminação, poder-se-ia desenvolver um desequilibrio hidroeletrolítico, com a } \\
\text { consequente desidratação e piora do seu Quadro clínico }\end{array}$ \\
\hline $\begin{array}{l}\text { Monitorar as eliminações urinárias e } \\
\text { intestinais diariamente }\end{array}$ & Esta intervenção visou avaliar a resposta da paciente face à terapêutica adotada \\
\hline $\begin{array}{l}\text { Inspecionar diariamente a } \\
\text { integridade da pele }\end{array}$ & $\begin{array}{l}\text { Por ser a paciente diabética e com mobilidade no leito e física prejudicada, a probabilidade de se desenvolverem } \\
\text { úlceras, a partir de pequenos ferimentos, é aumentada e esta conduta teve por objetivo minimizar estes riscos }\end{array}$ \\
\hline $\begin{array}{l}\text { Estimular a hidratação da pele, } \\
\text { principalmente nas extremidades dos } \\
\text { membros inferiores }\end{array}$ & $\begin{array}{l}\text { Conforme evidenciado no exame físico, a pele da paciente encontrava-se desidratada, o que facilita o aparecimento de } \\
\text { lesóes na pele e com isso, a hidratação da pele, através do uso de óleos e hidratantes, visa minimizar estes riscos }\end{array}$ \\
\hline $\begin{array}{l}\text { Orientar Quanto aos benefícios da } \\
\text { realização de atividades físicas } \\
\text { diárias }\end{array}$ & $\begin{array}{l}\text { Devido ao seu estilo de vida notadamente sedentário, fez-se necessário informá-la Quanto aos benefícios dos } \\
\text { exercícios físicos, até mesmo para amenizar a sua constipação }\end{array}$ \\
\hline $\begin{array}{l}\text { Estimular a deambulação por } 15 \\
\text { minutos }\end{array}$ & $\begin{array}{l}\text { deambulação auxilia a motilidade gastrointestinal, principalmente na liberação de flatos, além de ser um importante } \\
\text { meio para reduzir prejuízos na mobilidade física do indivíduo hospitalizado }\end{array}$ \\
\hline Auxiliar na deambulação & $\begin{array}{l}\text { Por ser uma paciente idosa, sem vontade expressa de deambular e da certa dificuldade para realização desta } \\
\text { atividade, foi importante auxiliar a mesma a deambular }\end{array}$ \\
\hline $\begin{array}{l}\text { Monitorar a glicemia capilar } \\
\text { sanguínea }\end{array}$ & $\begin{array}{l}\text { O paciente diabético necessita de um controle glicêmico rigoroso, a fim de monitorar e tomar as medidas cabíveis em } \\
\text { caso de hiper ou hipoglicemia. Desta forma, esta conduta foi essencial para a paciente, pelo fato da mesma utilizar } \\
\text { insulina para obter um controle glicêmico }\end{array}$ \\
\hline $\begin{array}{l}\text { Monitorar os sinais vitais, } \\
\text { principalmente a pressão arterial e o } \\
\text { pulso }\end{array}$ & $\begin{array}{l}\text { A monitorização frequente e rigorosa da pressão arterial são de fundamental importância, pois conforme identificado } \\
\text { durante a realização do histórico de enfermagem, a paciente é portadora de hipertensão arterial sistêmica (HAS). A } \\
\text { HAS, juntamente com a dislipidemia, também apresentada pela mesma, é fator de risco muito importantes, o Que } \\
\text { aumenta a propensão para o desenvolvimento de doenças cardiovasculares, como o IAM, e de doença } \\
\text { cerebrovasculares, como o AVE }\end{array}$ \\
\hline
\end{tabular}

Apêndice 2. Prescrições propostas com as respectivas justificativas. 